\section{Die Bedeutung der Prophylaxe für das Biofilmmanagement}

tursachen von gingivalen, parodontalen und dentalen Schädigungen. Ein umfassendes Biofilmmanagement betrachteten sie als wesentlichen Teil der parodontalen Infektionskontrolle.

Auch auf das Thema Patientenaufklärung und Motivationsstrategien für eine optimale Mundhygiene wurde eingegangen. Einer Meinung waren die Experten, dass die tägliche 3-fach-Prophylaxe dabei ein ideales Instrument zur Kontrolle des dentalen Biofilms darstellt. In diesem Zusammenhang betonte Prof. Dr. Rößler: „Es ist bekannt, dass gründliches Zähneputzen und die Reinigung der Zahnzwischenräume für eine gesunde Mundhöhle nicht immer ausreichen. Ergänzend zur mechanischen Zahnreinigung angewendet, können Mundspülungen mit ätherischen Ölen, wie z. B. Listerine, die schädlichen Bakterien beseitigen und zugleich die Neubildung von Belag zeitlich verzögern." Bei der anschließenden Diskussion mit den Fachpressevertretern antwortete Prof. Rößler auf eine Frage, was denn der der Vorteil der ätherischen Öle sei, dass die meisten Spüllösungen auf Wasserbasis konzipiert seien, der Biofilm dagegen sei aber lipophil. Durch die ätherischen Öle Thymol, Menthol, Eukalyptol und Methylsalicylat in Listerine kann die Struktur des Biofilms zersetzt werden. 